\title{
Réflexions sur la mémoire discursive
}

Reflexions on Discursive Memory

\section{Sírio Possenti}

Traducteur : Phillipe Enrico

\section{OpenEdition \\ Journals}

Édition électronique

URL : http://journals.openedition.org/aad/1200

DOI : $10.4000 /$ aad. 1200

ISSN : 1565-8961

Éditeur

Université de Tel-Aviv

Référence électronique

Sírio Possenti, «Réflexions sur la mémoire discursive », Argumentation et Analyse du Discours [En ligne], 7 | 2011, mis en ligne le 15 octobre 2011, consulté le 23 septembre 2019. URL : http:// journals.openedition.org/aad/1200; DOI : 10.4000/aad.1200

Ce document a été généré automatiquement le 23 septembre 2019.

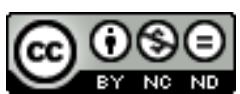

Argumentation \& analyse du discours est mis à disposition selon les termes de la licence Creative Commons Attribution - Pas d'Utilisation Commerciale - Pas de Modification 4.0 International. 


\title{
Réflexions sur la mémoire discursive
}

\author{
Reflexions on Discursive Memory
}

Sírio Possenti

Traduction : Phillipe Enrico

\section{NOTE DE L'ÉDITEUR}

Cet article a paru en portugais sous le titre « Slogans que se retomam » dans Possenti, Sírio (dir.). 2009. Questões para analistas do discurso (São Paulo : Parábola Editorial), pp. 127-140.

1 Les slogans politiques, et plus particulièrement ceux qui circulent lors des campagnes électorales, reprennent des slogans antérieurs, se construisent à partir d'énoncés courants d'ordre éthique et moral, ou encore sont fondés sur des lieux communs qui résument des idéologies de parti. Ils se situent donc dans un espace de relations intertextuelles et interdiscursives. Cette observation, qui n'est pas inédite, peut être aisément vérifiée : il suffit de comparer des campagnes électorales en examinant dans la presse, les archives, la mémoire « individuelle », etc., quels candidats ont été associés à tel ou tel slogan, ou encore quels candidats se sont réclamés de certaines vertus et comment ils ont stigmatisé leurs adversaires.

2 Un exemple notoire au Brésil est celui des slogans élaborés autour des termes " faire » et « voler » dans la campagne destinée à élire un gouverneur et un maire de São Paulo : en 2000 , une série de grandes affiches véhiculaient des énoncés comme «Erundina a fait X $(\mathrm{Y}, \mathrm{Z}$...) sans voler » (à la place de X, Y, Z... on pouvait lire « tant de maisons, tant d'écoles, tant d'hôpitaux, etc. »). Il n'y a là cependant rien d'évident, et l'objectif de cette réflexion consiste à se demander dans quelle mesure les slogans font appel à la mémoire, et s'il s'agit d'une mémoire discursive qui réside dans un rapport entre énoncés, ou plutôt d'une mémoire (ou d'un souvenir?) appartenant à des sujets, 
électeurs ou citoyens - ce qui, apparemment, situerait le problème dans le domaine de la cognition et/ou de la pragmatique.

3 La thèse que je souhaiterais défendre, du moins en ce qui concerne les campagnes électorales, est que la mémoire dont il y est question doit être quelque chose de plus qu'une machinerie discursive ou un fond d'archive, et cela dans la mesure où l'effet visé par la publicité est indissociable des reprises et associations (fussent-elles inconscientes et «imaginaires») qui sont réalisées par les électeurs et qui dépassent de ce fait la relation entre énoncés comme saisie par la mémoire discursive. Il s'agirait peut-être d'un phénomène de l'ordre de ce que signalent Pêcheux et Fuchs (1975:14) lorsqu'ils notent que « les processus discursifs [...] ne sauraient avoir leur origine dans le sujet. Néanmoins, ils se réalisent nécessairement dans ce même sujet ». Dans cette optique, il me semble que l'affirmation suivante de Bosi (1979: 333), qui résume la position que j'aimerais pouvoir justifier, est exemplaire : «Malgré tout ce que l'on doit à la mémoire collective, c'est l'individu qui se souvient ».

\section{Réflexions sur la question de la mémoire discursive}

\subsection{Un cas de figure : Casoy et Cardoso}

Dans la nuit du 7 décembre 1993, Boris Casoy s'entretenait avec le ministre de l'Économie de l'époque, Fernando Henrique Cardoso qui avait, dans l'après-midi, présenté un nouveau plan économique pour le Brésil. Le journaliste conclut l'entrevue en disant, avec un sourire ironique : «Malgré le fait que nous soyons vous et moi, Dieu vous entende ». Le ministre rit et remercia le journaliste.

5 Tous les téléspectateurs n'ont sans doute pas compris ce qui avait incité Boris Casoy à dire "malgré le fait que nous soyons vous et moi ", avant la formule rituelle "Dieu vous entende ». Mais beaucoup comprirent l'allusion. Pour ce faire, ils durent associer d'une façon ou d'une autre le commentaire de Boris Casoy à un autre événement discursif, qui avait eu lieu quelques années auparavant. En 1985, quand Fernando H. Cardoso était candidat à la mairie de São Paulo, eut lieu un de ces débats devenus quasi routiniers dans les élections ultérieures, pendant lequel les candidats répondent, entre autres, aux questions des journalistes. Boris Casoy, qui travaillait à l'époque au journal Folha de São Paulo, était présent et avait demandé à Fernando H. Cardoso s'il croyait en Dieu. La motivation immédiate de la question, dans un pays à électorat (majoritairement) catholique, venait du fait que Fernando $\mathrm{H}$. Cardoso avait la réputation d'être un intellectuel de formation marxiste, ce qui impliquait en principe son athéisme. Dans sa réponse, Fernando H. Cardoso tourna autour du pot, mais finit par avouer son athéisme. Pour de nombreux analystes politiques, cet aveu fut la raison principale de sa défaite au profit de Jânio Quadros.

$6 \quad$ Nul doute que Boris Casoy ait dit cela le 7 décembre 1993 pour s'être souvenu de la question qu'il avait posée au cours du débat de 1985. Il n'y a de même rien d'extraordinaire à postuler que cette connexion a été faite par les téléspectateurs qui s'intéressent à l'information politique (le fait de se référer à l'intérêt manifesté pour la politique oblige ipso facto à penser la mémoire comme n'étant pas purement "mentale » dans la mesure où cet intérêt en est la condition sine qua non, ce qui permet de dépasser ou d'abandonner l'hypothèse de quelque faculté mentale qui ne serait conditionnée par rien). Notons que, pour échapper à une conception étroite de la mémoire, on peut 
imaginer une explication alternative selon laquelle un membre de l'équipe du journaliste lui aurait suggéré de clore l'entrevue de cette manière. La fin de l'entrevue aurait pu être alors, non le résultat d'un souvenir personnel, mais l'effet d'une recherche dans des archives audio-visuelles - indiscutablement un type de mémoire, dans l'un des sens du terme. Cependant, même si cela était effectivement arrivé, il serait difficile d'expliquer sans aucun type de recours à la mémoire ou au souvenir le sourire de Fernando Henrique Cardoso, qui est dû certainement au fait que lui aussi associa instantanément les propos de Casoy à cette même scène de 1985. Cela étant, il n'est pas nécessaire qu'il y ait eu accord préalable entre les deux hommes sur ce qui allait se dire pendant l'entrevue, encore moins sur sa conclusion (envisager une telle hypothèse serait accorder un crédit excessif à la théorie de la conspiration).

7 Pour présenter les faits dans toute leur complexité, j'ajouterai que le 10 novembre 2000 le journal télévisé diffusé par le présentateur mit en valeur une visite de F. H. Cardoso, déjà président, dans l'Etat de Minas Gerais. Il était question de l'inauguration d'une usine, événement auquel le gouverneur de l'Etat ne se rendit pas parce qu'il ne voulait pas rencontrer F. H. Cardoso, même dans un cadre officiel. Durant la cérémonie, il y eut un échange tendu entre le représentant officiel du gouverneur et Cardoso qui, dans sa réponse (c'est ce qui nous intéresse ici), mentionna plusieurs fois Dieu, à partir de la citation d'une phrase de Juscelino Kubitschek : « Dieu ne m'a pas transmis le sentiment de la peur " (Kubitschek fut gouverneur de l'Etat de Minas Gerais [1951-1955] et président du Brésil [1956-1961]). À la fin du journal télévisé, Boris Casoy fit ce commentaire : « Monsieur le Président parle beaucoup de Dieu ces derniers temps ». Un léger sourire accompagna la remarque, que j'interprète comme un sourire de complicité à l'égard d'un certain nombre de téléspectateurs, ceux-là précisément qui comprirent l'allusion.

On pourrait se demander quelle importance attribuer au fait d'associer au commentaire du 10 novembre 2000 celui du 7 décembre 1993, et de relier ces deux commentaires, par un autre biais, à la question de Casoy à Cardoso lorsqu'il était candidat au poste de maire en 1985. La réponse en est que ces faits apparemment banals servent à illustrer un processus constitutif du fonctionnement des discours. J'aimerais avancer qu'il s'agit également du processus constitutif de la démarche interprétative à laquelle se livrent des lecteurs/auditeurs particuliers.

\section{Quelques considérations sur la mémoire}

9 Le développement qui précède montre que je ne souhaite pas identifier discours à discours immémorial ; c'est que, selon moi, un peu paradoxalement, il n'y a de sens qu'à partir du type de matériau que l'on a défini comme mémoire. Comme si, pour déloger le biologisme attaché à la mémoire, on avait dû éliminer tout type de mémoire à court ou moyen terme - c'est, je crois, ainsi que s'expriment les psychologues auxquels font en général appel les pragmaticiens.

En simplifiant quelque peu, on pourrait présenter les diverses conceptions de la mémoire auxquelles les spécialistes du langage se réfèrent, en les regroupant en trois grands courants. Dans le premier, on pourrait situer les psychologues ou neuropsychologues qui associent plus ou moins directement cerveau, pensée, mémoire et autres facultés (conscience, par exemple). Même quand ils n'oublient pas d'autres conditionnements, ils ne mentionnent pas, en général, des facteurs comme 
l'inconscient, l'idéologie ou même l'histoire. Pour beaucoup, cette position est nettement en déclin, et paraît difficilement défendable, du moins sous sa forme la plus radicale. Elle est considérée comme une étape historique dépassée dans ce domaine de recherche (comme on peut le voir, par exemple, chez Middleton et Edwards 1999 : 17).

11 En poussant à l'extrême cette conception, on en viendrait à supposer qu'un individu, indépendamment des facteurs sociaux qui l'affectent, peut, après un certain temps, se souvenir d'un événement particulier exactement comme il est arrivé. Il me semble que personne n'assume cette conception - qui est, cependant, mise sur le compte des "psychologues » dans des textes polémiques qui défendent des conceptions opposées: ainsi on lit dans Pêcheux (1999: 50) que la mémoire ne doit pas être entendue dans le sens directement psychologiste de "mémoire individuelle», et dans Courtine (1981: 52) que « ce que nous entendons par les termes de "mémoire discursive" est distinct de toute mémorisation psychologique du type de celle dont les psycholinguistes s'attachent à produire la mesure chronométrique ». On pourrait taxer cette conception de biologiste, plus que de psychologiste.

Une deuxième conception, nettement opposée à la précédente, se situe probablement à l'autre extrême. Bien qu'il y soit implicite que l'énonciateur est un sujet, il n'est pas toujours entendu que ce qu'il dit puisse être associé d'une certaine façon à un souvenir personnel distant, même " déformé » par le passage du temps, ou encore par le fait que le sujet "assista» à un événement à partir d'une position déterminée et le «mémorisa » ou le stocka dans un espace mental loin d'être vide. La métaphore la plus appropriée à cette conception serait peut être la mémoire informatique, non parce qu'elle est plus puissante ou plus "modulaire ", mais parce que ce qu'elle enregistre l'est dans un langage qui précède l'enregistrement et que, comme tout un chacun le sait, les ordinateurs n'ajoutent aucun détail " personnel » à l'événement enregistré, c'est-à-dire que rien ne peut y être considéré comme étant de nature psychologique. Je crois que des conceptions de la mémoire comme celles d'Achard et de Pêcheux entrent dans cette catégorie. De fait, pour Achard (1999), la mémoire est constituée par les présupposés (ou implicites) qui permettent l'émergence d'énoncés nouveaux (ils rappellent les préconstruits). Pour Pêcheux (1984: 262), la "mémoire doit être comprise non pas dans le sens directement psychologiste de "mémoire individuelle", mais aux sens entrecroisés de la mémoire mythique, de la mémoire sociale inscrite dans des pratiques, et de la mémoire construite de l'historien». Pour Courtine (1981), le domaine de la mémoire

est constitué par un ensemble de séquences qui préexistent [à une séquence spécifique], en ce sens que certaines formulations isolables dans la mise en place en séquences intradiscursives qui la [séquence] réalise [...] entrent [...] dans des réseaux de formulations à partir desquels seront analysés les effets que produit au sein d'un processus discursif l'énonciation d'une [séquence] déterminée (effets de reprise, de redéfinition, de transformation, mais aussi effets d'oubli, de rupture, de dénégation, de déjà dit).

La conception de Foucault est peut-être encore plus radicalement détachée de toute conception de la mémoire un tant soit peu mentaliste, tout au moins quand il s'agit du rôle de la mémoire dans le processus énonciatif. Pour Foucault (1969: 65), l'organisation du champ des énoncés comporte une division en plusieurs domaines, qu'il classe en "formes de succession", "formes de cohabitation" et "procédures d'intervention». C'est dans les formes de cohabitation qu'il inclut le «domaine de mémoire ", caractérisé (de façon sans doute surprenante) de la façon suivante : 
il s'agit des énoncés qui ne sont plus ni admis ni discutés, qui ne définissent plus par conséquent ni un corps de vérités ni un domaine de validité, mais à l'égard desquels s'établissent des rapports de filiation, de genèse, de transformation, de continuité et de discontinuité historique (1969: 78). ou plutôt, rien n'est personnel ni naturel, ce qui, dans ce cas, signifie que rien n'est purement «mental ». Plutôt que de se conformer simplement à l'idée que la mémoire est sociale, il est cependant nécessaire d'expliciter en quoi elle est sociale et comment elle se constitue comme telle, ou encore, de spécifier ses modalités sociales en expliquant pourquoi nous la nommons encore mémoire. J'avoue que, très souvent, lorsque je lis que la mémoire est sociale, historique ou discursive, j'ai la sensation qu'il s'agit de métaphores qui s'apparentent à celle de l'éponge analysée par Bachelard (1938 : 91-102), qui masquait et obscurcissait plus qu'elle ne résolvait les problèmes.

17 Sauf erreur, je crois qu'il serait utile de distinguer ce que l'on pourrait nommer « savoir » et ce qui pourrait s'appeler « mémoire d'une formation discursive », quelque étroite que soit la relation entre les deux. Les conceptions de la mémoire qui s'éloignent des positions d'Achard et de Pêcheux, pour ne pas citer celle de Foucault, caractérisent de façon adéquate ce que serait le savoir d'une formation discursive : les positions à partir desquelles un sujet peut énoncer. Mais je pense qu'il serait plus approprié d'imaginer que la mémoire est caractérisée par sa relation à des faits, et non par sa relation à des savoirs, c'est-à-dire en termes d'événements, et non en termes de restrictions « sémantiques » sur ce qui peut ou ne peut être dit. De cette façon, je pense que l'on pourrait, entre autres choses, situer d'un côté l'ensemble des énoncés qu'un sujet reprend, répète, reformule, etc. et, d'un autre côté, un ensemble d'événements (éventuellement, un événement singulier), qui, probablement pour de nombreuses raisons, fonctionnent comme une des conditions de production de tel énoncé déterminé ou comme élément à prendre en compte pour l'interpréter. Éventuellement, la formulation d'un énoncé peut en venir à fonctionner comme un événement (et non comme un élément de savoir), comme dans l'«affaire » Casoy-Cardoso : ce que Casoy a demandé à Cardoso et ce que ce dernier a répondu n'arrive pas à constituer (la part d') un savoir qui fonctionnerait comme restriction sur des énoncés possibles. Cela continue à être un épisode, un événement dont on se souviendra ou que l'on oubliera, ou plus encore, qui sera « mémorisable » ou " oubliable » en fonction des contraintes propres à une formation discursive. 

tente de proposer. Examinons ces exemples :

1) Le patient rencontre son analyste dans l'ascenseur et le salue: - Bonjour, Docteur. Comment vais-je?

On peut dire que le discours de ce patient émerge d'un savoir (stéréotypé, etc.) sur la psychanalyse, de la même manière que d'autres blagues émergent de "savoirs » relatifs à d'innombrables champs : ethnies, institutions, etc.

2) Un dessin humoristique de 1990 montre l'ex-ministre Erundina vêtue en médecin, auscultant les côtes de l'ex-ministre Eliseu Resende avec son stéthoscope. La légende est : « Dites $33 »$.

Il est inutile de chercher dans quelque discours que ce soit - c'est-à-dire dans les énoncés de quelque formation discursive caractérisée par certaines positions, par des savoirs, par des visions du monde, par des idéologies, etc. - le sens de la blague. Elle ne fonctionne que s'il y a mémoire de certains événements, dans le sens plus ou moins courant des termes de "mémoire» et d' "événement ». À savoir: si les sujets se souviennent que Resende était ministre de l'Économie sous le gouvernement d'Itamar Franco, que ses calculs lui faisaient dire que l'augmentation de la fonction publique ne pouvait dépasser les 17 \% (ce détail, à la vérité, pouvait même être oublié ou méconnu, il suffisait de se souvenir qu'il proposait une augmentation moindre que celle proposée par la ministre), et que Luiza Erundina était ministre de l'Administration dans le même gouvernement et proposait une augmentation de $33 \%$. Sans ces données, la caricature n'est qu'un simple dessin, peut-être la représentation d'une scène du quotidien, de la routine d'un cabinet. Évidemment, sont permises, et même nécessaires, d'autres associations avec de vraies consultations médicales.

21 En résumé, je crois que nous gagnerions en clarté si le point de départ pour distinguer savoir et mémoire était à peu près le suivant: un savoir serait caractérisé par un ensemble de restrictions sémantiques (comme proposé par Maingueneau [1984], par exemple) ; la mémoire se caractériserait par un ensemble de représentations de faits (des événements, principalement, mais aussi des énoncés, dans leur matérialité même, ou encore, des énoncés comme événements). De cette façon, par exemple, fait partie d'un savoir discursif (d'un certain discours de droite) le fait de dire que la gauche engendre le désordre ; un homme de droite peut le dire n'importe quand, comme on l'entend dans de nombreuses manifestations de campagnes électorales (le sujet n'a pas besoin de se souvenir de quoi que ce soit pour lancer cette affirmation): aucun énonciateur n'oublie une doctrine, ou un savoir. Mais il est possible d'« oublier » un fait particulier, un exemple que l'on «aimerait " mentionner, ou même la formulation exacte d'un proverbe ou d'une blague, ou une citation littérale, etc. En d'autres termes, le fait qu'un événement est toujours analysé ou appréhendé à partir d'une formation discursive ne l'assimile pas pour autant aux énoncés qui la caractérisent. Les gens peuvent se demander « qu'est-ce qui est arrivé ?» ou « qu'est-ce qu'il a dit? », mais pas «qu'est-ce que je pense des noirs / des femmes / du Parti des Travailleurs / des libéraux du Parti du Front Libéral? ». 


\section{La question de la mémoire discursive à l'aune du slogan électoral} déposséder encore plus radicalement le sujet énonciateur de toute activité discursive. Il peut paraitre correct d'analyser l'émergence d'un énoncé lié au savoir comme s'il dépendait peu du sujet et beaucoup de sa position, mais il est difficile de le faire quand il s'agit d'un événement. Le savoir est plus «anonyme " qu'un événement. Pour cette raison tout le monde comprend les blagues qui se fondent sur un certain type de savoir et beaucoup ne comprennent pas les blagues qui se basent sur des événements. Des énoncés de la campagne électorale de 2000 à Campinas (ville de l'Etat de São Paulo) peuvent aussi servir à exemplifier cette distinction. Le slogan du PSDB (Parti de la Sociale-Démocratie Brésilienne), quoiqu'il contienne des ingrédients «éthiques » et, par conséquent, doctrinaires, essayait de se référer à un fait, un mandat antérieur : le slogan «Campinas veut faire revenir le sérieux » présupposait en effet qu'il y avait déjà eu du sérieux à la mairie de Campinas. Quant au slogan du PPB (Parti Progressiste du Brésil), il était franchement doctrinaire, prenant simplement appui sur un certain savoir («je bats ferme! [mon pouls]»), que l'on peut évidemment associer aux fondements doctrinaires de ce parti ; mais pour que ce slogan fonctionne, il n'est pas nécessaire que celui-ci ait pu montrer à quel rythme exact battait le pouls du parti.

Les slogans mentionnés plus haut peuvent affiner cette distinction : dans sa campagne pour gagner la mairie de São Paulo, aux élections de 2000, Luiza Erundina rappela aux électeurs, dans une série de grandes affiches, ce qu'elle avait fait lors du précédent mandat. Les affiches, on l'a dit, véhiculaient des énoncés comme «Erundina a fait $\mathrm{X}$ sans voler ». Il est possible d'effectuer une distinction entre les nombreux éléments de cet énoncé qui relèvent du savoir et de la mémoire. Il y a des éléments de doctrine matérialisés dans la référence au «faire » (dans la politique brésilienne, pour le moins, cet élément est fondamental, et s'oppose à des points de vue comme « faire plutôt que parler ", avoir la "réunionite ", etc.); il y a des éléments de mémoire tant dans les chiffres mentionnés que dans le fait de se souvenir que le candidat adverse avait volé de l'argent. Ou alors, si Luiza Erundina agit au lieu de parler, on peut voter pour elle, même si l'on n'est pas de gauche - il faut préciser qu'elle se présenta aux élections municipales de 1966 avec le slogan «Le Parti des Travailleurs qui agit », pour contrer d'une certaine manière les accusations selon lesquelles le PT ne faisait rien, parce qu'il tardait considérablement à prendre ses décisions ou parce que les militants du parti empêchaient les élus d'agir.

J'aimerais présenter un autre exemple et esquisser son analyse pour tenter d'éclaircir plus avant le problème. En bref, les faits sont les suivants. Au début de l'année 2000, suite à des événements plus ou moins choquants dont la violence était le principal ingrédient surgit dans cette même ville un mouvement pour la paix : à Rio de Janeiro l'attaque à main armée d'un autobus avait provoqué la mort de plusieurs personnes, parmi lesquelles une jeune femme assassinée dans des circonstances mal élucidées, suite à une intervention incompétente de la police, alors que tout laissait penser que le dénouement serait moins tragique. Le slogan de ce mouvement, qui aurait dû générer une mobilisation de masse importante dans bien des villes du pays, était : «Assez! Je veux la paix ».

Argumentation et Analyse du Discours, 7 | 2011 

pourrait être résolu), la question se pose de savoir s'il y a ou non un "propriétaire » du slogan, et, formulée de la sorte, la question se perd dans les dédales des droits d'auteur. Mais elle n'en est pas résolue pour autant, si ce n'est pour les avocats. Il y a pour le moins deux autres aspects importants à explorer. L'un d'eux a à voir avec le lieu d'où émerge le discours. Le slogan original naît - en simplifiant quelque peu - d'une position qui stipule que la violence est un mal; plus précisément, d'une évaluation selon laquelle elle est devenue insupportable, a passé toutes les bornes. C'est ainsi que se justifie le « assez ». La solution proposée est la substitution de la paix à la violence. On peut remarquer que la violence n'est pas mentionnée dans le slogan (en cela, il y a un effet de sens très particulier, qu'il vaudrait la peine de discuter : est-il de savoir ou de mémoire ?). Nous savons que celui-ci est un slogan (à l'intérieur d'un mouvement) qui combat la violence puisque tous les moyens de communication nous informent que c'est bien de cela qu'il s'agit. De la sorte, il n'est pas nécessaire de dire « Assez de violence!». Le slogan formule le problème comme si nous étions dans un contexte de guerre. Tel est l'effet de sens du mot « paix », son antonyme.

Ainsi, la première des deux questions porte sur le fait de savoir comment (au-delà des subtilités juridiques) le slogan de Tuma fonctionne à partir du slogan de l'Institut « Je suis [du côté] de la paix ». Je crois que tout le monde s'accorderait à dire que le deuxième slogan est construit à partir du premier, sauf d'éventuels interprètes payés pour défendre Tuma devant un juge, qui adopteraient une position insoutenable sur le plan lexical, affirmant qu'il n'y a pas la moindre relation entre " paix » et " Tuma », de façon à montrer qu'il n'y a pas de plagiat. Plus encore : il apparaît que la différence entre " paix » et « Tuma » est exactement ce que l'on doit effacer, c'est-à-dire que les publicitaires de l'ex-commissaire entendent précisément poser, ici et maintenant, une équivalence entre "paix » et «Tuma » afin que ceux qui en auraient assez de cette violence votent Tuma, son antonyme hic et nunc. Plus que la résonance d'une voix dans une autre, l'effet « visé » est d'identifier Tuma et paix avec la fin de la violence que lui seul pourrait faire advenir. De ce point de vue, le plagiat est évident. Paradoxalement, les publicitaires "veulent » qu'il y ait plagiat, qu'un slogan soit de fait équivalent à l'autre. C'est pourquoi la défense ne se fonde pas sur l'exposition de la différence, mais sur le caractère anonyme et universel du slogan. 
ne deuxième question est : dans quelle mesure s'approprie-t-on le slogan en prenant pour base l'hypothèse que pourrait s'effectuer le rapprochement espéré entre le mouvement pour la paix et la campagne du candidat? Autrement dit, dans quelle mesure fait-on appel à la mémoire des électeurs et non à « leur » savoir ? On ne doit pas sous-estimer la perspicacité des professionnels de la publicité : ils savent que les gens «se souviennent » des faits et des discours (même en tant que faits...) sur la violence, que ce soit pour parler de ses excès ou pour proposer des solutions. Quelle aurait été l'efficacité du slogan que l'équipe de Tuma s'est approprié, si le rapport entre ce slogan et le slogan des mouvements pour la paix n'avait pas été plus ou moins évident pour tous?

On pourrait prétexter que l'association entre les deux slogans se fait plus ou moins inconsciemment, que cette association est "libre ", au sens freudien - c'est-à-dire, qu'elle ne l'est pas... Elle peut l'être, mais je ne manquerai pas de citer une interprétation de Reboul au moment où il rapproche le fonctionnement des slogans de celui des facéties : étant donné que ce que dit le slogan, il le dit, à la manière des facéties, en peu de mots, Reboul ajoute que, dans les deux cas, cette économie donne une sensation de pouvoir, «le plaisir d'une découverte» (1975:61-2). L'efficacité de ce slogan dépend moins du fait que le rapprochement entre le slogan de Tuma et celui du mouvement pour la paix est l'effet d'une mémoire discursive, que du fait que le destinataire de la campagne effectue une découverte (l'association entre deux slogans, l'équivalence entre Tuma et paix) et se perçoit ainsi comme un électeur intelligent et non manipulé.

\section{Evénements historiques et événements discursifs : l'éclairage de Foucault}

30 Ce qui a été conceptualisé plus haut gagne à être considéré à la lumière de la notion d'événement telle que l'élabore Foucault (1972). Explicitant la "contribution» à l'histoire des études liées au structuralisme, il affirme que certaines méthodes ont permis de «donner un sens nouveau à la notion d'événement» (1994: 276). Le prix à payer fut que l'histoire dut cesser d'être une discipline

grâce à laquelle la bourgeoisie montrait d'abord que son règne n'était que le résultat, le produit, le fruit d'une lente maturation et que, dans cette mesure-là, ce règne était parfaitement fondé, puisqu'il venait de la nuit des temps; ensuite, la bourgeoisie montrait que, puisque ce règne venait du fond des temps, il n'était pas possible de le menacer par une révolution nouvelle (1994:272).

31 L'histoire doit être surtout comprise « comme l'analyse des transformations dont sont effectivement susceptibles les sociétés. Les deux notions fondamentales [...] ne sont plus le temps et le passé, mais le changement et l'événement » (273).

Pour rendre explicite le nouveau sens de l'événement, Foucault fait référence à une étude des archives commerciales du port de Séville au 16e siècle: "tout ce qui concerne l'entrée et la sortie des bateaux, leur nombre, leur cargaison, le prix de vente de leurs marchandises, leur nationalité, l'endroit d'où ils venaient, l'endroit où ils allaient » (276). Comme on peut le voir, « l'objet de l'histoire n'est plus donné par une sorte de catégorisation préalable en périodes, époques, nations, continents, formes de culture [...]» (ibid.) et ne consiste plus à déchiffrer quelque chose comme le développement de l'Espagne ; l'objet de la recherche historique est d'établir un certain

Argumentation et Analyse du Discours, 7 | 2011 
nombre de relations. L'historien "n'interprète plus le document pour saisir derrière une sorte de réalité sociale ou spirituelle qui se cacherait en lui ; son travail consiste à manipuler et à traiter une série de documents [...] » (277).

De telles études, dit Foucault, peuvent faire apparaître « des événements qui autrement ne seraient pas apparus»(ibid.). Dans l'histoire traditionnelle, explique-t-il, on considérait que les événements étaient ce qui était connu, visible, directement identifiable, et le travail de l'historien consistait à chercher leur cause et leur sens. L'histoire sérielle, par contre, permet de faire apparaître des couches d'événements dont certains sont visibles et connus, y compris de leurs contemporains, mais endessous desquels se trouvent d'autres événements, invisibles, imperceptibles et d'un type complètement différent.

Foucault fournit didactiquement divers exemples d'événements que révèle ce type d'étude. L'entrée et la sortie d'un bateau sont des événements connus et que l'on peut reconstituer. Mais la baisse ou l'augmentation des prix est également un événement. Et sous ces derniers il y en a encore d'autres, qui ne sont pas perçus de la même façon par leurs contemporains, mais dont ils avaient néanmoins une certaine conscience. En dessous d'eux, encore d'autres, comme l'inversion d'une tendance, le point à partir duquel une courbe économique qui avait été croissante devient stable ou décroît - et qui peut ne pas être perçue par les contemporains (277-78). Les économistes euxmêmes ne savent pas, dit Foucault, si « un point d'arrêt dans une courbe économique signale un grand renversement général de la tendance. [...] C'est à l'historien de découvrir cette couche cachée d'événements diffus [...] qui, finalement, déterminent, et profondément, l'histoire du monde » (278). Au-delà de ces types d'événement, Foucault en mentionne d'autres : la croissance de la population, la croissance considérable de la quantité de protéines absorbée par la population européenne au $19 \mathrm{e}$ siècle, extrêmement importante pour la santé et la longévité. Foucault dit de ces événements qu'ils sont des faits beaucoup plus importants que le changement de constitution ou qu'un passage de la monarchie à la république (ibid.).

Cette notion d'événement permet peut-être de repenser le problème de la mémoire. Je dirais que son effet le plus immédiat serait d'accepter que, relativement à certains événements, il n'est pas absurde de supposer que les habitants d'une ville en aient conscience. La division que j'ai proposée ci-dessus entre événements et savoirs pourrait être subsumée par une variété de types d'événement. J'accepterais volontiers cette hypothèse. Ce qui me paraît actuellement étrange, dans l'AD, c'est l'exclusion totale de ce qui pourrait ressembler au souvenir. Celui-ci est cependant tout à fait acceptable pour peu que l'on postule qu'il existe divers types d'événements et, par conséquent, divers types de mémoire. L'AD, typiquement, pense le problème de la mémoire sous des angles que les théories psychologiques ne prennent pas en compte. Ce qui lui manque dès lors, selon moi, est de prendre en compte et d'examiner à travers son propre point de vue les phénomènes auxquels s'intéressent les psychologues.

S'il existe des types d'événements historiques dont on a une parfaite conscience et d'autres qui nous échappent, l'analogie avec divers types d'événements discursifs pourrait nous conduire à repenser certains aspects de la conception du sujet dans l'AD. Ne devrait-on pas ainsi, par exemple, rediscuter les conceptions que l'on se fait de l'oubli? 


\section{BIBLIOGRAPHIE}

Achard, Pierre. 1984. « Mémoire et production discursive du sens », Achard, Pierre, Max-Peter Gruenais \& Dolores Jaulin (éds). Histoire et linguistique (Paris : Editions MSH), 235-242

Bachelard, Gaston. 1938. La formation de l'esprit scientifique: contribution à une psychanalyse de la connaissance (Paris : Vrin)

Bosi, Ecléa. 1979. Memória e sociedade : lembranças de velhos (São Paulo : Companhia das Letras)

Courtine, Jean-Jacques. 1981. Analyse du discours politique : le discours communiste adressé aux chrétiens (Paris : Didier)

Foucault, Michel. 1969. L'archéologie du savoir (Paris : Gallimard)

Foucault, Michel. 1972. « Revenir à l'histoire », Dits et écrits II (Paris : Gallimard), 268-281

Maingueneau, Dominique, 1984. Genèses du discours (Bruxelles : Mardaga)

Middleton, David \& Derek Edwards. 1990. Memoria compartida : la naturaleza social del recuerdo y del olvido (Barcelona : Paidós)

Pêcheux, Michel. 1984. « Rôle de la mémoire », Achard, Pierre, Max-Peter Gruenais \& Dolores Jaulin (éds). Histoire et linguistique (Paris : Editions MSH), 261-268

Pêcheux, Michel. 1999. « Papel da memória », Achard, Pierre (éd.). Papel da memória (Campinas : Pontes), 49-57

Pêcheux, Michel \& Catherine Fuchs. 1975. « Mises au point et perspectives à propos de l'analyse automatique du discours ", Langages 37, 70-88

Reboul, Olivier. 1975. Le slogan (Bruxelles : Complexe)

\section{RÉSUMÉS}

Ce travail problématise la notion de "mémoire discursive" en analysant des événements à propos desquels il ne semble pas approprié de postuler une mémoire «individuelle et psychologique ». Il pose qu'il importe de prendre en compte l'activité des sujets impliqués jusque dans leur dimension individuelle, en reprenant l'idée que «malgré tout ce que l'on doit à la mémoire collective, c'est l'individu qui se souvient ». L'hypothèse qui sous-tend l'article se fonde sur la séparation entre savoir et mémoire: le premier se rapporte au sens des discours, la seconde aux discours comme événements.

This paper deals with discursive memory, analyzing events for which it does not seem appropriate to postulate that memory is "individual and psychological." However, it is necessary to consider that some type of activity performed by the subject, be it on the individual level, is involved and has to be considered. The underlying hypothesis behind this paper is the separation between knowledge and memory, the former referring to the meaning of discourses, and the latter to discourses as events. 
INDEX

Mots-clés : discours, événement, mémoire, savoir

Keywords : discourse, event, knowledge, memory

\section{AUTEURS}

\section{SÍRIO POSSENTI}

Universidade Estadual de Campinas 